\title{
Memoria por procuración y vindicación de la genealogía femenina en la narrativa de Maryse Condé
}

Recibido: 15/01/2015

Aceptado: 13/04/2015

\section{RESUMEN:}

El propósito de este artículo es ofrecer una lectura de la novela biográfica Victoire, les saveurs et les mots (2006), de la antillana Maryse Condé, en clave memorística y de género. Nos serviremos de la noción de 'post-memoria' o 'memoria por procuración', definida por la canadiense Marianne Hirsch, para tratar de la búsqueda identitaria del 'yo' creador. Por otra parte, queremos demostrar que este ejercicio narrativo-memorístico vehicula la reivindicación de una genealogía femenina y materna, lo que hermana el discurso de Condé con el de otras autoras francófonas contemporáneas, como la argelina Assia Djebar.

PALABRAS CLAVE: mujer, post-memoria, género, diferencia, genealogía, matriarcado, francofonía, narrativa.

\section{ABSTRACT :}

The purpose of this paper is to present a reading of the biographical novel Victoire, les saveurs et les mots (2006), from Antillean writer Maryse Condé, focused in two capital aspects : memory and gender. We will use the notion of 'post-memory', defined by the canadian narrator Marianne Hirsch, to study the creator's identity search in this story. We also want to show that Victoire, les saveurs et les mots, like most stories from Algerian writer Assia Djebar, contents a strong vindication of a feminine and maternal genealogy.

KEY-WORDS: women, post-memory, gender, difference, genealogy, mothering, French-speaking communities, narrative. 
En su novela Victoire, les saveurs et les mots (2006), la escritora antillana Maryse Condé (Pointe-à-Pitre, isla de Guadalupe, 1908) viene a (re)vindicar el bagaje artístico, filosófico, social y cultural femenino recibido en los márgenes de la escuela, la familia y sus historiografías oficiales -tanto individuales como nacionales-: correas de perpetua transmisión de los cánones intelectuales patriarcales.

Se trata, más concretamente, de la reconstrucción de la biografía de su abuela materna, Victoire Quidal, mestiza originaria de la isla guadalupeña de Marie-Galante. Empleamos aquí a conciencia el término 'reconstrucción', pues Condé acomete la tarea de relatar la vida de su antepasada adoptando y adaptando a las exigencias narrativas de la tercera persona el precepto básico de la denominada 'autoficción': todo relato literario es (auto)biográfico, toda (auto)biografía es ficcional ${ }^{1}$. La fabulación y la autorreferencialidad vendrán, de este modo, a reparar todo eventual silencio histórico: "Imaginons, c'est tout ce qui nous reste"2.

De forma análoga, en el célebre relato $L a$ nuit du récit de $F a-$ tima, de la argelina Assia Djebar ${ }^{3}$ (Cherchell, Argelia, 1936), escuchamos las voces de la contadora y la escuchante refiriéndose, confundidas, a la performación como imperativo existencial: “Mais le récit est exigeant. C'est meme ce qui reste, seulement ce fil d'argent ou de noir ébène, luisant dans la longue nuit..." Este relato de Djebar se publicó por vez primera en 1980, reeditándose en 2002 en el volumen de relatos Femmes d'Alger dans leur appartement. Apenas cuatro años separan, por lo tanto, tan

1 Recordemos que se le atribuye el bautismo de la noción de 'autoficción' a Serge Doubrovsky, quien sería el primero en emplear este neologismo, en 1977, en su novela Hilos. Vid. Doubrovsky, Serge. Fils, París, Gallimard, 2001. p. 160.

2 Condé, Maryse. Victoire, les saveurs et les mots, París, Mercure de France, 2006

3 Djebar, Assia. Femmes d'Alger dans leur appartement, París, Albin Michel, 2002.

4 Djebar, A., Femmes..., p. 37. 
significativa reedición de la aparición de la novela de Condé que nos ocupa. Ambas obras, como tendremos ocasión de ir viendo a continuación, comparten, no obstante, similitudes que van más allá de su relativa contemporaneidad ${ }^{5}$.

En Victoire..., los límites entre realidad y ficción se difuminarán hasta desaparecer en un tan voluntario como voluntarioso ejercicio de "memoria por procuración"'6. Los postulados de tal 'pacto ${ }^{7}$ o juego memorístico-creativo le son explicitados al lector, para su aceptación activa, desde el incipit mismo de la novela. Se abre ésta con una muy relevante cita de un ensayo sobre escritura y literatura firmado por el intelectual francés Bernard Pingaud8: "Il devient indifférent que je me souvienne ou que j'invente, que j'emprunte ou que j'imagine" ${ }^{\prime \prime}$.

Detengámonos un instante en los términos 'memoria por procuración' o 'post-memoria', tal y como los define Marianne

5 Recomendamos vivamente, sobre el parentesco literario existente entre estas dos grandes narradoras francófonas contemporáneas y otras (a saber: la también argelina Farida Belghoul y la camerunesa Calixthe Beyala), el revelador estudio comparativo de Martine Fernándes, publicado en 2007. Vid. Fernándes, Martine. Les écrivaines francophones en liberté : Farida Belghoul, Maryse Condé, Assia Djebar, Calixthe Beyala, París, L'Harmattan, 2007. Por otra parte, para profundizar en la obra de Assia Djebar contextualizada, aconsejamos el remarcable trabajo de Marta Segarra sobre las novelistas francófonas del Magreb. Vid. Segarra, Marta. Leur pesant de poudre: romancières francophones du Maghreb, París, L'Harmattan, 1997.

6 Retomamos aquí la noción y la terminología acuñadas por la narradora canadiense Marianne Hirsch para referirse al conocimiento indirecto, mediante testimonios; y al esfuerzo consciente del sujeto por recuperar la(s) historia(s) silenciada(s) que le explican, esto es, la idea de la identidad y la memoria como fabulación o construcción, búsqueda y conquista personales. Vid. Hirsch, Marianne. Family frames. Photography, narrative and postmemory, Cambridge, MA: Harvard University Press, 1997.

7 Vid. Lejeune, Philippe. Le pacte autobiographique, París, Seuil, 1975.

8 Vid. Pingaud, Bernard. Les Anneaux du manège. Écriture et littérature, París, Gallimard, 1992.

9 Condé, M., Victoire..., p.11. 
Hirsch. El lugar de la fotografía, como indica el título de su obra Family frames. Photography, narrative and postmemory, es proeminente en la construcción del concepto. Hirsch toma en sus estudios como principal punto de partida el Holocausto para observar la problemática de su conocimiento indirecto en las generaciones posteriores al mismo. Concluye entendiendo por "postmemoria" la relación que con los hechos en cuestión establecen aquellos que suceden a los testigos: sus descendientes o las "post-generaciones"10. Su experiencia de esos hechos se construye, así, sobre discursos narrativos de diversa índole: oral, como ocurre en La nuit du récit de Fatima; fotográfico-documental, como en el caso de Victoire ${ }^{11}$...

En efecto, se recurre a menudo en Victoire... a fotografías familiares, cartas y recortes varios de la prensa local guadalupeña para llevar a cabo la (re)contrucción de la figura de las ancestras. Estos elementos se constituyen en motores de la narración. Según los episodios, reactivan el relato, legitiman o desautorizan sus posibles, desencadenan la fabulación y la (auto)ficción...

Le journal "Le Nouvelliste " célébra la réussite de Jeanne au brevet supérieur par un article vibrant intitulé: "Négresse en avant! En avant toute! " (...) Les photos de ces pionnières parurent dans "Femmes en devenir ", organe qu'on peut qualifier de féministe. Dans la revue mensuelle "La Guadeloupe de demain"12.

Así las cosas, este retrato novelado de Victoire Quidal pretendería constituirse como 'contrarretrato', si se nos permite la

10 Hirsch, Marianne. <<The Generation of Postmemory $>$, in Poetics today, Columbia University, 2008, nำ29, p. 103.

11 Resulta de sumo interés el trabajo de Lourdes Carriedo López, Mª Dolores Picazo y Ma Luisa Guerrero (2013) sobre el empleo del motivo fotográfico en la narrativa contemporánea en lengua francesa y, más concretamente, en la obra de Annie Ernaux (vid. Bibliografía).

12 Condé, M., Victoire..., p. 191. 
expresión, del rostro oficial que a lo largo de las décadas habría conservado la protagonista en la familia de nuestra autora. ¿A qué nos referimos con la expresión 'rostro oficial'? A la imaginería de la mestiza bastarda que engendrará, a su vez, mestizos bastardos; la paupérrima empleada doméstica, analfabeta, creolófona $-\mathrm{y}$, en consecuencia, incivilizada ${ }^{13}{ }^{3}$, sumisa, humillada, animalizada, etc.: máximo exponente de la intranscendencia $y$, en suma, ancestral vergüenza para cualquier familia de burgueses 'bon nègs' que se precie de serlo.

Recordemos a este respecto que las expresiones 'bon nèg' o 'bel nèg' (en francés, 'bon nègre' o 'bel nègre') fueron comunes, durante la primera mitad del s.XX, entre las poblaciones criollas de Guadalupe, Martinica, La Dominica, San Martín y Guyana. Por oposición a la etiqueta peyorativa 'ti nèg' ('petit nègre'), significaban, en primer lugar, el orgullo de un color de piel sin mezcla; amén de una cierta conciencia de élite socioeconómica e intelectual surgida como reacción a las élites blancas, francófonas y metropolitanas, en cuyo espejo, paradójicamente, los 'bel nègs' no cesaban de mirarse.

Maryse Condé se propone demostrar la peligrosa ambigüedad moral de todos estos estereotipos raciales, con el fin de visibilizar, dignificándola, la figura injustamente silenciada de su abuela materna, que formaría parte del folclórico conjunto de los 'ti nègs': "Pour nous tous, cette grand-mère à l'étrange couleur fut à moitié imaginaire. Un esprit. Un fantôme. Couché dans la nuit du temps longtemps"14.

En el caso de La nuit du récit de Fatima, contemplamos una idéntica voluntad de contrarretratar a la narradora ${ }^{15}$ protagonis-

13 ...le français, cette arme clé sans laquelle les portes de la civilisation demeurent closes (Condé, M., Victoire..., p. 205).

14 Condé, M., Victoire..., p. 262.

15 “Elle soupire, hésite (...) puis, en se rasseyant sur la natte, le chapelet à la main, et ses yeux dans le vague, elle redevient narratrice" (Djebar, A., Femmes..., p. 28). 
ta, Fatima, madre de la escritora aquí reducida a la condición de escuchante ${ }^{16}$ y mera transcriptora: "Fatima qui raconte, va enfin oser se raconter" ${ }^{\prime 17}$.

Se hace así patente, en ambos relatos, una profunda búsqueda identitaria del sujeto creador. Por decirlo de otro modo: tal afán por demostrar el valor de la figura de la 'ancestra' como piedra fundacional del linaje artístico familiar, testimonia una intensa indagación íntima acerca del propio lugar y la propia naturaleza. En estos términos lo explica la propia Condé -su 'yo' artístico, para ser más exactos- en uno de los incisos o metalepsis que abundan en la novela: “Ce que je veux, c'est revendiquer l'héritage de cette femme qui apparemment n'en laissa pas. Établir le lien qui unit sa créativité à la mienne"18. Djebar, por su parte, se detendrá más bien en la naturaleza liberadora de esta búsqueda identitaria, que indefectiblemente pasa por la apropiación femenina de la palabra: “...je ressens combien parler sur ce terrain devient (...) d'une façon ou d'une autre une transgression" ${ }^{\prime 19}$.

Con la explicitación de estas motivaciones, hace su aparición implícita en ambos discursos la noción de 'genealogía femenina', que tanto ha ocupado a las pensadoras de los feminismos en general y del denominado 'feminismo de la diferencia sexual' ${ }^{20}$ en particular. Ya en el primer cuarto del pasado siglo, la novelista inglesa Virginia Wolf, en Una habitación propia (1929), reflexionó

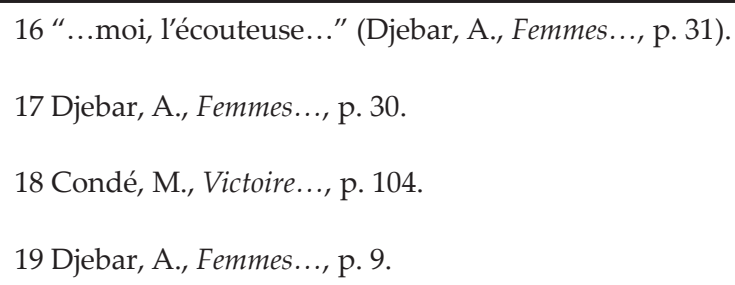

20 Destacan los trabajos de la pionera francesa Lucie Irigaray (1985 y 1987), que prestó especial atención a los referentes históricos, filosóficos y divinos en femenino; o, como muy bien lo resume la española Mâa-Milagros Rivera Garretas, al esclarecimiento de una "genealogía que da sentido y placer al estar en el mundo en femenino" (Vid. Rivera Garretas, Milagros. La diferencia sexual en la historia, Valencia, Publicaciones de la Universidad de Valencia, 2005). 
sobre este concepto de la red de conocimientos y referencias propias a la mujer versus el bagaje masculino, dirigiéndose de este modo a las autoras contemporáneas:

Porque, si somos mujeres, miramos el pasado a través de nuestras madres. Es inútil buscar ayuda en los grandes escritores, por más que una pueda buscar en ellos placer (...). El peso, el paso, la zancada de la mente de un hombre son demasiado distintos de los suyos para que ella pueda sacar de él algo sustancial con éxito. El simio es demasiado distante para ser imitable ${ }^{21}$.

La genealogía intelectual femenina, por lo tanto, tiene su base afectiva en la familia como "matronado" 22 y en la madre como piedra angular. Al decir 'madre', entiéndase también "abuela, bisabuela materna, hijas" ${ }^{23}$ : toda antepasada, en fin, reducida u olvidada por causa del exilio forzado de la mujer a la familia y, en consecuencia, la genealogía masculina del padre-marido.

En ese sentido, consideramos que, tanto en Victoire... como en La nuit du récit de Fatima, entra en juego el sentimiento de orfandad intelectual femenina y de rebeldía frente a la exclusividad de la genealogía masculina familiar que les ha sido legada a nuestra autoras. Nos parece importante discernir que no se trata tanto de una negación del lenguaje y el conocimiento patriarcales, sino de su apertura hacia un sistema transmisor de cultura y pensamiento doblemente sexuado y, por ello, más justo. Se trata, retomando las palabras de Djebar, de desenterrar el lenguaje " $\mathrm{du}$ corps voilé, langage à son tour qui si longtemps a pris le voile" 24 .

21 Wolf, Virginia. Un cuarto propio y Horas, trad. de María-Milagros Rivera Garretas, Madrid, Alianza Editorial, 2003.

22 Traducimos aquí el término 'matronage', antónimo de la dinámicas relacionales de poder que moviliza el 'patronage' o patriarcado, tal y como lo emplean las italianas Lucía Ferrante, Maura Palazzi y Giana Pomata (1988).

23 Irigaray, Lucie. Sexes et parentés, París, Les Éditions de Minuit, 1987.

24 Djebar, A., Femmes..., p. 8. 
El establecimiento del mencionado lazo de génesis creativa femenina se funda sobre una necesaria redefinición de las problemáticas nociones de 'arte' y 'cultura'. Una reescritura de ambos conceptos que permita acercar, para comprenderlas, las figuras de Fatima y Djebar, de Victoire y su nieta Condé... Dediquémosle un poco más de atención a este último binomio. La primera trabajó toda su vida como empleada del hogar: al servicio de los Walberg, una familia de 'blancs pays' o 'bekés' ${ }^{25}$, durante más de dos décadas; y para su hija Jeanne, en sus últimos años. La segunda, Maryse Condé, primogénita de dos de los primeros maestros negros de la isla de Guadalupe, Jeanne Quidal y Auguste Bocoulon ${ }^{26}$, es escritora. ¿Qué lazo creativo puede, pues, unir a ambas mujeres? Ninguno, en apariencia.

La respuesta, como avanzábamos, reside en la flexibilización de la idea de creación y sus implicaciones. No sólo la cultura y el arte tradicionalmente entendidos como tales lo son. También lo diminuto y lo doméstico, como en el particular que nos ocupa, pueden serlo. Victoire Quidal fue una muy reputada cocinera, cuya fama se extendió por todos los rincones de Guadalupe. Las mejores familias de la isla intentaron, en vano, contratarla. Condé encuentra en este aspecto el antecedente artístico que buscaba en su linaje. Mediante una potente red metafórica que identifica cocina con escritura, se honra el talento, la inteligencia, la obra y la dignidad de la abuela:

25 En las Antillas francesas, se denominan 'blancs pays' o 'békés', según las islas, a las etno-clases blancas y burguesas, de origen metropolitano.

26 Recordemos que el apellido de soltera de nuestra autora es Bocoulon. Condé es, en realidad, el apellido de su primer marido y padre de sus hijos, el actor guineano Mamadou Condé. Se casaron en Guinea en 1959 y, tras su divorcio en 1964, Maryse se trasladó a Gana con sus hijos. Desde allí viajaría posteriormente a Londres, París, su Guadalupe natal y Estados Unidos, donde reside en la actualidad. 
Cuisiner, c'était son rhum Père Labat, sa ganja ${ }^{27}$, son crack, son ecstasy. Alors, elle dominait le monde. Pour un temps, elle devenait Dieu. Là aussi, comme un écrivain ${ }^{28}$.

La iletrada Victoire Quidal se nos introduce así como una artista pura o, por retomar la terminología convencional de la historiografía artística contemporánea, una artista 'bruta'29. La denominación 'arte bruto' o 'arte marginal' engloba las producciones de creadores con escasa o nula formación académica, a veces aquejados de enfermedades mentales $y$, en definitiva, outsiders de muy variada condición. Artistas ajenos a los cauces academicistas o canónicos de la cultura. Artistas que, como la propia Victoire entre fogones, acusan los procesos creativos desde la total extrañeza crítica, es decir, instalados en el terreno de lo absolutamente natural por intuitivo e innato.

En este orden de cosas, serán siempre los observadores externos -el público- de este tipo de obras quienes las califiquen de artísticas, manteniendo sus autores una postura de humildad rayana en el anonimato:

Victoire n'appréciait pas tout ce bruit autour de sa personne. C'est à regret qu'elle confiait à Anne-Marie le secret de ses compositions culinaires afin que celle-ci les baptise et les fasse imprimer. Comme un écrivain dont l'éditeur décide du nom, de la couverture, des illustrations de l'ouvrage, c'était en partie se dessaisir de sa création ${ }^{30}$.

27 De 'ganjah', término utilizado por las rastafari para referirse al cannabis o la marihuana, muy consumida en las Antillas.

28 Condé, M., Victoire..., p. 123.

29 El primer teórico del "art brut" sería, a mediados del s. XX, el artista francés Jean Dubuffet. Vid. Dubuffet, Jean. L'Art Brut préféré aux arts culturels, Paris, Galerie René Drouin, 1949.

30 Condé, M., Victoire..., p. 123. 
En nuestra novela, ese rol analítico lo desempeñan aquellos personajes que, invitados a la mesa de los Walberg o los Bocoulon, tienen el honor de probar los magníficos manjares ideados y ejecutados por Victoire. También, más allá de la rígida codificación de las recepciones sociales burguesas, por las calles y comercios populares de Pointe-à-Pitre, ejercerá la crítica entusiasta de la obra culinaria de Victoire el personaje de la costurera Jeanne Repentir. Victoire tiene por costumbre regalarle, como prueba de su amistad, elaborados dulces de frutas tropicales que invariablemente la conducen a reaccionar como sigue:

-Tu es un poète, un poète, disait Jeanne Repentir, mordant ces petites merveilles de ses dents gourmandes. Tu ne le sais pas, mais tu vaux tellement mieux que ta fille (p. 247).

Maryse Condé, como ya vemos desde el título mismo de la novela, toma en ella lo culinario como metáfora de la creación, en general, y de la creación literaria, en particular. No resulta en absoluto arbitraria esta elección. Se trata de una identificación que ya encontrábamos, por ejemplo, en el s. XVII, en la obra de la poeta novohispana Sor Juana Inés de La Cruz, que en su célebre carta de respuesta a Sor Filotea dejó escrito: "Si Aristóteles hubiera guisado, más hubiera escrito" ${ }^{\prime 31}$. Más recientemente, autores como Daniel Cassany, docente de la Universidad de Barcelona y autor del manual de narratología La cocina de la escritura, también han explotado con éxito el símil (1999, reed. 2004).

La cocina engrosa esa lista no escrita de actividades relativas al hogar y las dinámicas de cuidados que la tradición pretende exclusivamente femeninas. Actividades invisibles, denostadas: grilletes domésticos de la mujer. Maryse Condé, por virtud de la mencionada identificación simbólica cocina-escritura, subvierte 
esta connotación negativa y consigue imbricar lo físico o manual con lo intelectual, elevar lo menudo a la categoría de sublime. La cocina pasa entonces de ser yugo para la mujer a artefacto de expresión, insumisión, rebeldía y libertad; además de un ejemplar antecedente artístico en la familia, de incalculable valía para las generaciones venideras:

Il me plait, quant à moi, que ma grand-mère demeure secrète, énigmatique, architecte inconvenante d'une libération dont sa descendance a su, quant à elle, pleinement jouir ${ }^{32}$.

Asistimos de esta forma a un emotivo proceso de procuración de la memoria o, lo que es lo mismo, como ya sabemos, de procuración y esclarecimiento del poliedro de la propia identidad. La nieta se (auto)explica gracias al precedente creador que supone la abuela cocinera. A lo largo de la novela, además, veremos cómo Victoire acusa todas las fases y todos los conflictos típicos del autor literario... Escollos a los que Maryse Condé, en su ya larga trayectoria como narradora, ha debido sin duda enfrentarse. Se produce, por tanto, una suerte de trasvase discursivo, quedando a menudo íntimamente imbricados, cuando no confundidos, los discursos indirectos libres del 'yo' de la autora y de la supuesta protagonista del relato.

Victoire, decíamos, escenifica e ilustra a la perfección las principales problemáticas de la creación literaria. Tratemos de profundizar en esta cuestión. Partamos, para ello, de un interrogante capital: ¿cuál parece ser la peor de las vivencias posibles para un escritor? Convengamos en dar por respuesta la no-escritura, es decir, el silencio. Puede éste ser en mayor o menor medida autoimpuesto, como medida de protesta pasiva o auto-exilio; ser fruto de una crisis vital, un período de inspiración en barbecho o de la tan temida sequía artística...

32 Condé, M., Victoire..., p. 318. 
Victoire retrouvait l'odeur de la couenne du porc roussi, de la volaille farcie, de l'agneau braisé. Des cives. De l'ail. Des épices. Mais elle ne créait, n'inventait rien de nouveau. Elle ne faisait qu'indiquer de vieilles recettes à cette valetaille docile, plus habituée à bâcler des colombos qu'à parfaire des exploits culinaires. Comme un romancier qui réutilise sans vergogne les ficelles de ses anciens best-sellers (...) Quel écrivain produit chef-d'oeuvre après chef d'oeuvre ?33

Estos últimos propósitos de la narradora nos recuerdan sobremanera a los de la propia Maryse Condé en una de las entrevistas que concediera, en los tempranos años 90, a su amiga y estudiosa de su obra Françoise Pfaff. Al interesarse Pfaff por la heterogeneidad de la producción literaria de Condé, ésta acude resuelta a desmentir la premisa mayor, llegando a declarar: "We always write the same book" ${ }^{\prime \prime}$.

Mas regresemos ahora al problema del silencio creativo. Puede asimismo venir condicionado por cualquier otra modalidad de factores externos y ser, en consecuencia, un imperativo instaurado mediante dinámicas varias de dominación-sumisión. Estamos hablando, claro está, de censura:

Je me demande ce que signifiait pour Victoire le fait de ne plus s'approcher d'un potajé. Ne plus marier les saveurs, les couleurs. Ne plus respirer l'odeur des épices. Ne plus être Dieu.

Cette situation est comparable à celle d'un écrivain que des circonstances indépendantes de sa volonté tiennent éloigné de son ordinateur. Quel supplice! Comment lutter contre la terrible sensation d'inutilité qui l'envahit alors? ? $^{35}$

33 Condé, M., Victoire..., p. 295.

34 Pfaff, Françoise. Entretiens avec Maryse Condé, París, Khartala, 1993, p. 76.

35 Condé, M., Victoire..., p. 196. 
La subordinación intelectual respecto al censor presenta, según los contextos, matices y variaciones relacionales. Para nuestra narradora, las casas editoriales, por ejemplo, no necesariamente constituyen órganos inocentes de censura, pues con demasiada frecuencia privilegian la lógica colectiva de las leyes mercantiles frente a la individualidad de sus escritores. En la novela que estudiamos, este papel censor de la editorial lo desempeñan los hombres blancos para quien Victoire trabaja y su hija:

Pour rétablir la communication entre elles, Victoire dut se conformer à ses goûts, comme un auteur des Éditions de Minuit qui se piquerait d'écrire pour la Collection Harlequin ${ }^{36}$.

No nos parece ilegítimo escuchar aquí la voz de una Maryse Condé que ha alcanzado su máxima madurez y éxito internacional como narradora. Desde esa segura posición de independencia, se permite por fin denunciar ciertos abusos de los que se sintió quizás víctima en su juventud literaria. Este tema también lo abordaba, desde la perspectiva periodística, Pfaff en su libro de conversaciones con Condé. Más precisamente, en una charla a propósito del paradójico éxito de la saga maliense Ségou (la única obra de Condé que nacería, al parecer, por un encargo de Daniel Radfort, el editor de la casa parisina Robert Laffont), deseoso de emular el éxito de la archiconocida Raíces de Alex Haley... ${ }^{37}$

Victoire se trouva, comme un temps chez Anne-Marie, dans la position d'un écrivain forcé d'honorer des commandes d'éditeur. Très vite, son travail lui pèse, l'insupporte, devient corvée. Car la cuisine, comme

36 Condé, M., Victoire..., p. 313.

37 Pfaff, F., Entretiens..., p. 48. 
l'écriture, ne peut s'épanouir que dans la plus totale liberté et ne supporte pas les contraintes ${ }^{38}$.

La reparación histórica de la figura de la abuela Quidal pasa, como venimos argumentando, por su visibilización digna en tanto que creadora -sujeto activo- y no musa -sujeto pasivo u objeto-. Resulta capital, llegado este punto, integrar el carácter polifacético de su actividad artística, que no se circunscribió únicamente al espacio hasta ahora estudiado, el de la cocina-escritura, sino que también se desarrolló en otros ámbitos.

Consideremos, primeramente, el potencial dramatúrgico de Victoire. A saber: su presencia escénica en un sombrío, rígido y profundamente determinista decorado social. Centrémonos, a fin de cuentas, en "la femme qu'elle représentait" 39 . A pesar de su analfabetismo y de la incomodidad que el baile social le produce, Victoire se desenvuelve en él con corrección y actúa, nunca mejor dicho, mimetizándose con los ambientes de los salones burgueses donde su hija Jeanne la fuerza a figurar. Victoire se presta con docilidad a este sacrificio, deseosa de complacer las aspiraciones de ascenso social e intelectual de su hija: "Sa fille serait instruite. Elle se sacrifierait pour cela" ${ }^{40}$. Esta maternal auto-inmolación de Victoire pronto dará sus frutos:

Le 19 mai 1906, Jeanne fut la première fille noire à être admise avec la mention "Excellence" au concours qui ouvrait les portes du pensionnat de Versailles ${ }^{41}$.

38 Condé, M., Victoire..., p. 240.

39 Condé, M., Victoire..., p. 13.

40 Condé, M., Victoire..., p. 85.

41 Condé, M., Victoire..., p. 167. 
Una vez más, el paralelismo entre los relatos de Condé y Djebar parece evidente. En La nuit du récit de Fatima, gracias al sacrificio de los padres de la protagonista, ésta podrá comenzar, con seis años, una fulgurante aunque brevísima escolarización en una idealizada escuela republicana francesa: "Moi, première diplômée des filles dites 'indigènes' de la région" ${ }^{42}$.

Por otro lado, y regresando a Victoire..., cabe subrayar el hecho de que ésta (recordemos: mujer sola, mestiza, creolófona, sin instrucción y empleada doméstica) lleve, en un plano sentimental, una vida sorprendentemente libre para lo que su complicada condición podría augurar. Durante años, gozará de una activa vida amorosa y sexual, además de globalmente satisfactoria, con su patrón Boniface Walberg. Su fortaleza de carácter y su irreductible actitud vital, harán de Victoire, a pesar de todo, una mujer pionera en el arte de ser libre o, mejor dicho, en 'el arte de aprender a ser libre'. Por ello, su hija Jeanne la definirá de cara a las generaciones futuras en los siguientes términos: "Elle ne savait ni lire ni écrire. Pourtant, c'était un poto-mitan, une matador !"43.

Otra dimensión complementaria de la naturaleza artística de Victoire Quidal, que en esta novela se (re)vindica, la conformarían su exquisita sensibilidad musical, su sentido del ritmo y su oído privilegiados, patentes desde su más tierna infancia:

Dès que la musique s'éleva, Victoire s'approcha de l'appareil à le toucher. Elle resta clouée en place, fascinée par la lente rotation de l'aiguille. Quand la mélodie s'arrêta, elle se mit à piétiner, elle si douce : -Mizik! Mizik!44

\footnotetext{
42 Djebar, A., Femmes..., p.36.

43 Condé, M., Victoire..., p.18.

44 Condé, M., Victoire..., p.37.
} 
Maryse Condé reclama asimismo la rehabilitación de su ascendiente desde la óptica de su honda sapiencia connatural. Rechaza de todo punto su representación menguada, restricta al cliché de la criada ignorante... Pues, ¿qué es, en realidad, la sabiduría? ¿Dónde termina el conocimiento legítimo y da comienzo el vasto páramo de la incultura? Desde luego, no en el nombre ni el rostro de Victoire Quidal, parece respondernos a esto Maryse Condé. No. Porque Victoire Quidal fue, además de un genio indiscutible de la gastronomía, una mujer emocionalmente inteligente, docta en remedios naturales y caseros, erudita en cantos y danzas populares, versada en literaturas orales... Y poseedora, por encima de todo, de los atávicos saberes mujeriles que, a modo de advertencia, a través de los siglos, encomiendan las madres a sus hijas:

Elle lui en parlait beaucoup, des hommes, depuis qu'un an plus tôt, elle avait vu son sang. Elle lui contait leur scélératesse insondable. Leur traîtrise irrepressible. Leur irresponsabilité foncière ${ }^{45}$.

En este sentido, resulta pertinente mencionar que no es Victoire el único actante femenino del relato usufructuario y transmisor del capital femenino. Otros personajes vienen a completar esta genealogía femenina re-descubierta, presentándosenos en íntimo contacto con la(s) divinidad(es) y la naturaleza: celadoras-mediadoras de sus secretos... Es el caso, por ejemplo, de la costurera Repentir, de quien ya hemos tratado, que acostumbra a tener sueños premonitorios: "Elle avait vu ce moment en rêves et ses rêves ne mentaient jamais" ${ }^{\prime 4}$. Igualmente lo ilustra la bruja Bilé:

45 Condé, M., Victoire..., p. 41.

46 Condé, M., Victoire..., p. 245. 
Léonora Bilé, une kongo, savante en l'art des feuilles, sortait chaque jour de Trianon pour masser son corps amaigri avec de l'huile de coco dans laquelle avait macéré l'écorce de fagarier ${ }^{47}$.

Es frecuente, tanto en la narrativa de Condé como en la de Djebar, una estrecha identificación de estos personajes femeninos mediadores con lo invisible (el más allá, lo sobrenatural, la magia, lo onírico...) con la noche como momento privilegiado de la vida y, por ende, la palabra. El papel de la "noche créole" 48 y esta palabra nocturna como factores de desarrollo de la identidad cultural en los relatos de Maryse Condé ha sido estudiado con detalle, en la Universidad de Murcia, por Isaac Cremades, a cuya tesis doctora ${ }^{49}$ remitimos al lector.

Gracias a la resistencia y al inconsciente activismo feministas que, en muy diversos ámbitos y de muy diversos modos, vienen ejerciendo a lo largo de la historia mujeres anónimas (equiparables a estos personajes literarios-literalizados de Fatima, Repentir, Bilé y, por supuesto, Quidal), hoy resulta posible escribir, como Maryse Condé lo hiciera en esta novela de 2006, que "au tout début du $X X^{\mathrm{e}}$ siècle, la vie commença de changer pour les femmes" ${ }^{\prime \prime}$.

47 Condé, M., Victoire..., p. 65.

48 Cremades, Isaac: <<La noche "créole" en los relatos de Maryse Condé: desarrollo del imaginario y de la identidad cultural $\gg$, Estudios románicos, Murcia, Universidad de Murcia, 2013, vol. 22, pp. 29-42.

49 Cremades, Isaac. Oralidad e identidad femenina en la obra narrativa de Maryse Condé. Dir.: Antonia López Pagán, Murcia, Universidad de Murcia, Dpto. de Filología Francesa, Románica, Italiana y Árabe, 2014.

50 Condé, M., Victoire..., p. 213 


\section{Bibliografía}

Braidotti, Rosi et alii. Historia y fuente oral, Valencia, Publicaciones de la Universidad de Valencia, 1991.

Carriedo, Lourdes; Picazo, Dolores; Guerrero, Ma Luisa: $<<$ Fotografía versus escritura en "L'usage de la photo" de Annie Ernaux $\gg>$, Entre escritura e imagen. Lecturas de narrativa contemporánea, Bruselas, PIE Peter Lang, 2013.

Cassany, Daniel. La cocina de la escritura, Barcelona, Anagrama, 1999.

Condé, Maryse. Victoire, les saveurs et les mots, París, Mercure de France, 2006.

Cremades, IsaAc. Oralidad e identidad femenina en la obra narrativa de Maryse Condé. Dir.: Antonia López Pagán, Murcia, Universidad de Murcia, Dpto. de Filología Francesa, Románica, Italiana y Árabe, 2014.

Cremades, Isaac: <<La noche "créole" en los relatos de Maryse Condé: desarrollo del imaginario y de la identidad cultural $\gg$, Estudios románicos, Murcia, Universidad de Murcia, 2013, vol.22, pp. 29-42.

De la Cruz, Sor Juana Inés. Respuesta a Sor Filotea, Barcelona, Laertes, 1979.

Djebar, Assia. Femmes d'Alger dans leur appartement, París, Albin Michel, 2002.

Dubuffet, Jean. L'Art Brut préféré aux arts culturels, Paris, Galerie René Drouin, 1949.

Doubrovsky, Serge. Fils, París, Gallimard, 2001.

Ferrante, Lucía et alii. Ragnatele di rapporti, Torino, Rosenberg\&Sellier, 1991.

Fernándes, Martine. Les écrivaines francophones en liberté : Farida Belghoul, Maryse Condé, Assia Djebar, Calixthe Beyala, París, L'Harmattan, 2007. 
Hirsch, Marianne. Family frames. Photography, narrative and postmemory, Cambridge, MA: Harvard University Press, 1997.

Hirsch, Marianne: <<The Generation of Postmemory $>>$, Poetics today, Columbia University, 2008, no29, pp. 103-128.

Irigaray, Lucie. Sexes et parentés, París, Les Éditions de Minuit, 1987.

IRIgARAy, Lucie. El cuerpo a cuerpo con la madre. El otro género de la naturaleza. Otro modo de sentir, trad. de Mireia Bofill y Ana Carvallo, Barcelona, La Sal, 1985.

Lejeune, Philippe. Le pacte autobiographique, París, Seuil, 1975.

Pfaff, Françoise. Entretiens avec Maryse Condé, París, Éditions Karthala, 1993.

Pingaud, Bernard. Les Anneaux du manège. Écriture et littérature, París, Gallimard, 1992.

Rivera Garretas, María-Milagros. La diferencia sexual en la historia, Valencia, Publicaciones de la Universidad de Valencia, 2005.

Segarra, Marta. Leur pesant de poudre : romancières francophones du Maghreb, París, L'Harmattan, 1997.

Wolf, Virginia. Un cuarto propio y Horas, trad. de María-Milagros Rivera Garretas, Madrid, Alianza Editorial, 2003.

Marta Asunción Alonso Moreno

UNIVERSIDAD DE EXTREMADURA 
